\title{
Símbolos de informação ao público voltados ao contexto pandêmico
}

\section{Public Information Symbols Focused on the Pandemic Context}

Mariana S. Lukasinski, Isabela A. Ciuneck, Eduardo S. Zabloski, Ana Carolina S. Costa, Marcel P. Pauluk

pictogramas, ISO, pandemia

\begin{abstract}
Este artigo apresenta o desenvolvimento de pictogramas relacionados ao contexto pandêmico para inclusão na norma internacional ISO 7001. O projeto foi realizado através da seleção de 12 mensagens informacionais, de um levantamento dos pictogramas com mensagens semelhantes já existentes, da elaboração e design de novos pictogramas de acordo com a norma ISO 22727, da realização de testes de julgamento dentro do grupo de pesquisa, em seguida de um teste de compreensibilidade com o público de acordo com a norma ISO 9186, a fim de validar os resultados obtidos. Como resultado, a maioria dos pictogramas apresentaram respostas corretas, assertivas e similares e estão aptos para submissão à ISO 7001.
\end{abstract}

pictogram, ISO, pandemic

This article presents the processes involved in the design of pictograms related to the current pandemic context for inclusion in the ISO 7001 international standard. The project was carried out through the selection of 12 informational messages, a survey of the pictograms with similar existing messages, the elaboration and design of new pictograms according to ISO 22727, the application of judgment tests within the research group and, then a comprehensibility test with the general public, following ISO 9186, aiming at the validation of the results obtained. At the end of the process, most pictograms presented correct, assertive and similar answers and were able to be submitted to ISO 7001.

\section{Introdução}

No dia 11 de março de 2020, a Organização Mundial da Saúde (OMS) declarou estado de pandemia de COVID-19 (World Health Organization, 2020). Esse cenário exigiu mudanças de comportamento da sociedade a fim de evitar a disseminação do novo vírus, como usar máscaras e evitar aglomerações. Essas novas ações precisaram ser adotadas pela população, gerando uma demanda por símbolos gráficos de informação para comunicar e orientar práticas de prevenção contra o COVID-19. Curitiba | Brazil | 2021 
A norma internacional ISO 7001 - Símbolos de Informação ao Público ${ }^{1}$ especifica os pictogramas $^{2}$ a serem utilizados para a informação do público em todos os locais onde este tem acesso. Como esta é a norma adotada pelo Brasil (NBR ISO 7001: 2018) e por grande parte dos países do mundo, suas diretrizes e especificações foram utilizadas neste trabalho de iniciação científica, que por sua vez faz parte do projeto Pictogramas: História, Design e Normalização (Pauluk, 2020), da Universidade Federal do Paraná.

Foi realizado um levantamento de quais conceitos e ações poderiam ser relevantes para submissão à norma ISO 7001 através da ABNT/CEE-168 - Comissão de Estudo Especial de Símbolos Gráficos. Por conseguinte, foram selecionadas 11 mensagens relacionadas ao contexto pandêmico, como lavar as mãos, evitar aglomeração de pessoas, usar máscara, etc. e ainda uma mensagem relacionada ao crescente desenvolvimento tecnológico de aplicativos, amplificada pelo mesmo contexto (parada de veículos de aplicativo). Esses pictogramas visam atender às demandas sociais e científicas de precaução contra o COVID-19, assim como futuras pandemias.

\section{Perspectiva Teórico-Metodológica}

O contexto pandêmico introduziu novos protocolos de saúde pública e o reforço de práticas já conhecidas. Uma grande variedade de pictogramas foram produzidos ad hoc e utilizados por corporações públicas e privadas para comunicar estas mensagens. Entretanto, para que a compreensão desses símbolos seja efetiva, é necessário que haja uma padronização, garantindo clareza, consistência e uma melhor assimilação das mensagens. A ISO - Organização Internacional de Normalização (International Organization for Standardization, no original) é a responsável pelo estabelecimento das diretrizes que coordenam essas padronizações.

O uso dos pictogramas como um instrumento de comunicação com o público remonta ao desenvolvimento, no início do século XX, do Isotype - International System of Typographic Picture Education, de Otto Neurath, com intuito de criar uma linguagem visual internacional (Lupton, 2011), e expande-se até os dias de hoje em resposta ao processo de globalização. Diversas organizações ao redor do mundo criaram seus próprios repertórios de pictogramas, muitas vezes com projetos de baixa qualidade (Bakker, 2013). Apoiando-se nos primeiros esforços de padronização do uso de pictogramas, a ISO criou em 1970 um comitê técnico especializado para organizar e definir as diretrizes de padronização de conteúdo e formas dos símbolos gráficos, o ISO/TC 145.

\footnotetext{
${ }^{1}$ Símbolos Gráficos: termo utilizado em todas as normativas da ISO relacionados a pictogramas.

2 Pictogramas são símbolos gráficos que representam ações, objetos ou conceitos (Farias, 2003, p. 71).
} 


\section{Desenho da Pesquisa}

Este trabalho foi realizado através de processos iterativos e não-iterativos de design (Duarte et al., 2009) e seguindo a norma ISO 22727 , das diretrizes necessárias para o desenvolvimento de símbolos gráficos de informação ao público. O projeto desenvolveu-se em etapas: $\mathrm{Na}$ primeira delas (ideação), foram feitas simultaneamente a revisão bibliográfica a partir de trabalhos acadêmicos e de normas relevantes da ISO, objetivando compreender a temática e o estado da arte acerca do assunto e a identificação das necessidades ocasionadas pelo contexto pandêmico através do levantamento de palavras-chave ou breves descrições das mensagens de informação ao público necessárias.

$\mathrm{Na}$ segunda etapa (criação), foram realizados estudos iniciais de soluções para as mensagens definidas na etapa anterior. Durante essa etapa, foram levantadas diretrizes de projeto, pesquisados pictogramas semelhantes ou relacionados já em uso e criadas diversas versões e modificações dos símbolos gráficos (Holmes, 2001), através de discussões em grupo para que cada uma das mensagens possuíssem ao menos duas versões compreensíveis.

Figura 1: Processo de desenvolvimento das alternativas para "Desinfetar as mãos".

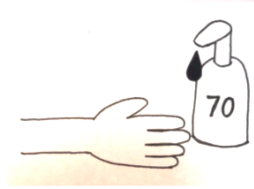

$\rightarrow$
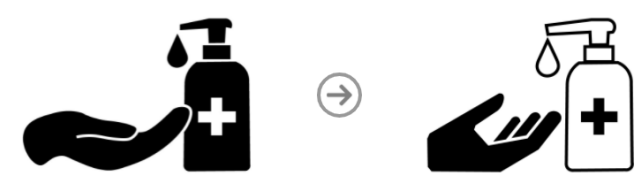

$\rightarrow$
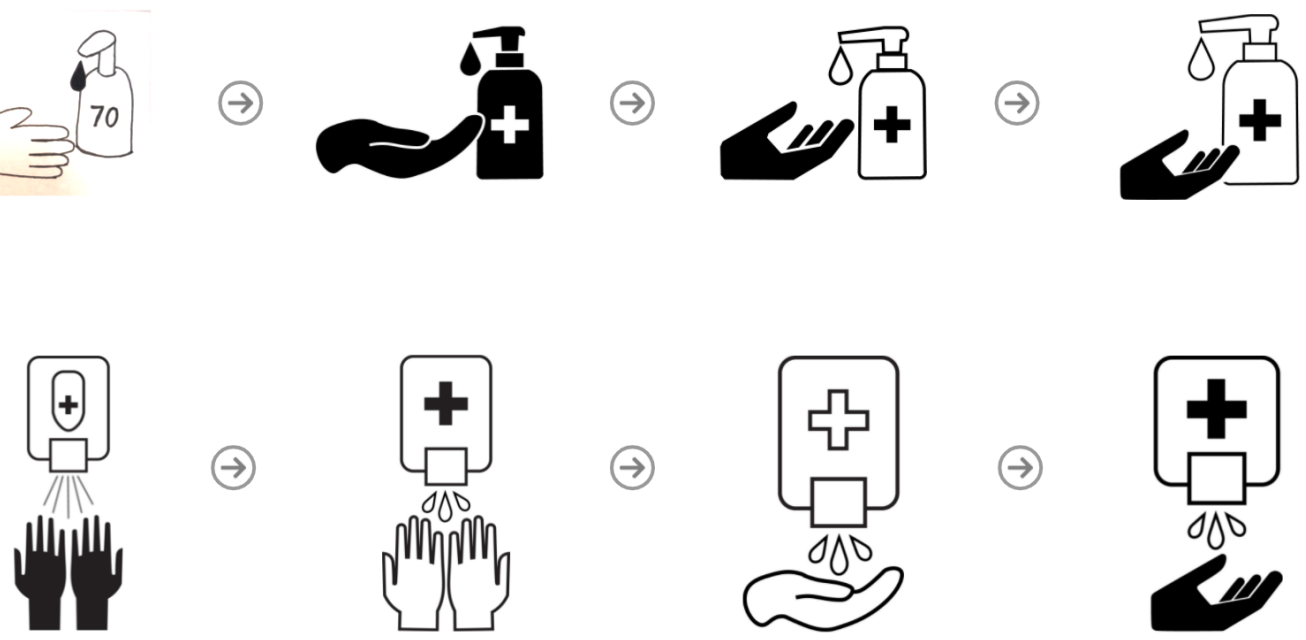

Na terceira etapa (avaliação), realizou-se testagens a partir das versões selecionadas para cada mensagem, totalizando 24 pictogramas (Tabela 1). Foram realizados 2 testes de compreensibilidade de acordo com as prerrogativas da ISO 9186 - Símbolos gráficos: Métodos de teste. Parte 1: Método para testes de compreensibilidade. Os pictogramas foram expostos sozinhos e dentro do contexto onde poderiam ser encontrados, e duas perguntas foram feitas ao usuário: "O que significa este pictograma?" e "Qual a ação deve ser feita ao ver esse pictograma?" (Figura 2). Através de formulário digital, foram coletadas 110 respostas, e dessas, 109 foram contabilizadas como válidas. 
Figura 2: Exemplo de página do formulário de compreensibilidade.

\section{Pictograma 1 de 12}

Observe o pictograma abaixo. A imagem ao lado representa um tipo de local onde você poderia encontrá-lo: mercados, lojas, transporte público etc.
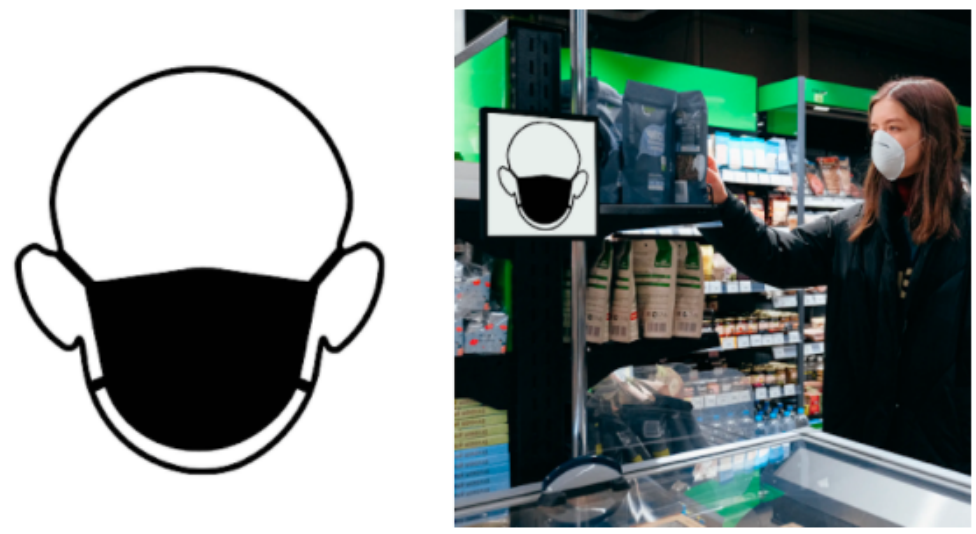

O que esse pictograma representa? *

Sua resposta

Qual ação deve ser realizada ao ver este pictograma? *

Sua resposta

Voltar Próxima

\section{Resultados e Discussões}

A ISO 9186 orienta que as respostas obtidas sejam categorizadas em 5 categorias: correta, errada, errada com significado oposto à correta, não soube responder, e nenhuma resposta. Para isso, foram analisados os contextos das respostas, levando em conta a aproximação com o referente proposto para cada pictograma. Respostas completamente descontextualizadas foram consideradas erradas.

As alternativas foram aprovadas conforme a compreensão dos usuários referente à ação e ao significado, com exceção de "Usar Máscara" e "Não Compartilhar Alimentos", pois as diferentes versões apresentaram resultados quase idênticos e foram, portanto, analisadas pelo grupo e julgadas de acordo com o melhor contexto. Na tabela 1, os dados obtidos são expostos de maneira resumida e aproximada. 
Tabela 1: Dados resumidos da tabulação realizada através da testagem.

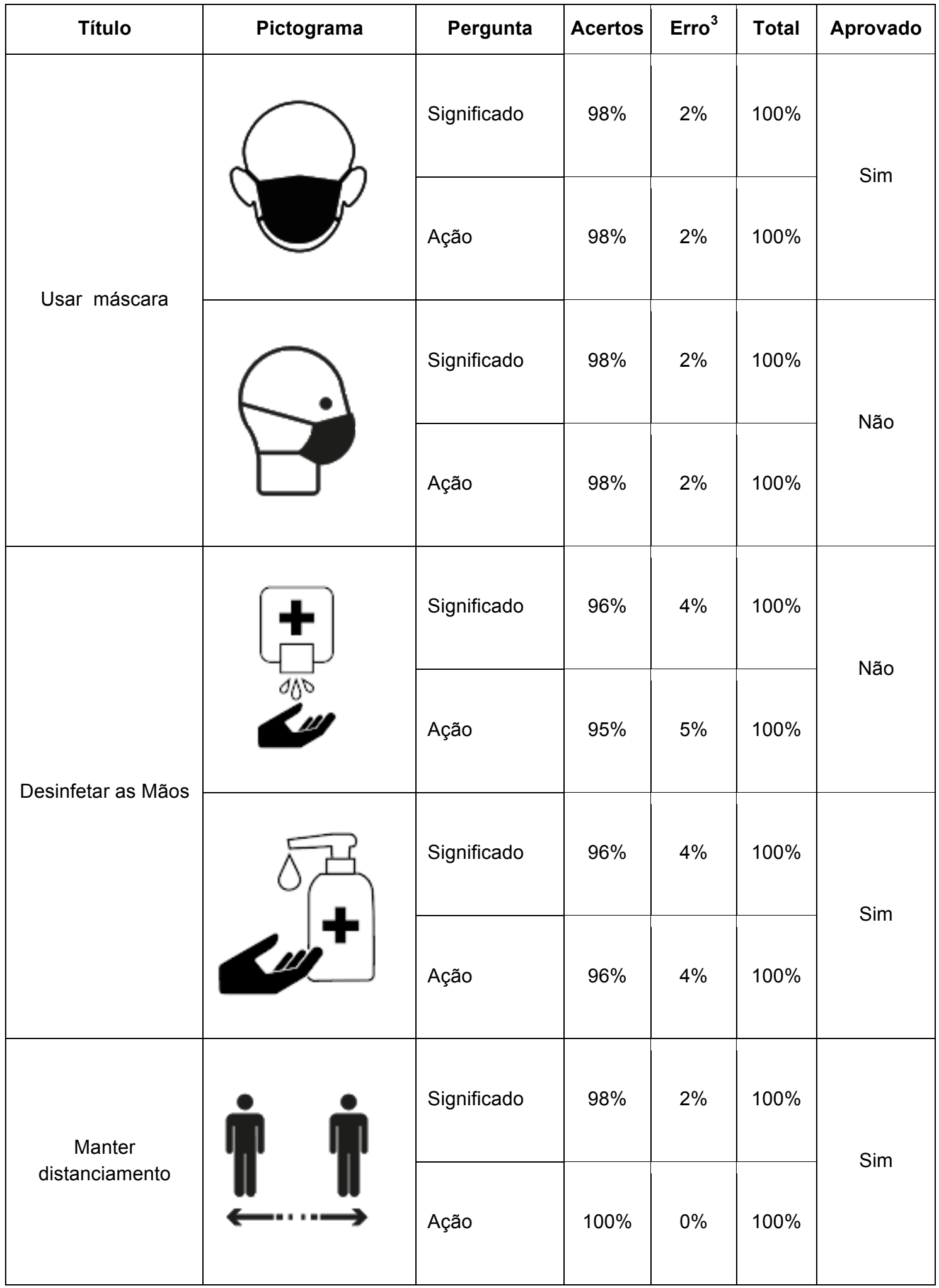

\footnotetext{
${ }^{3}$ Erros são a somatória de três categorias de respostas conforme a tabulação indicada pela ISO 22727: "Não saber", "resposta errada" e "resposta errada sendo o oposto da correta".
} 


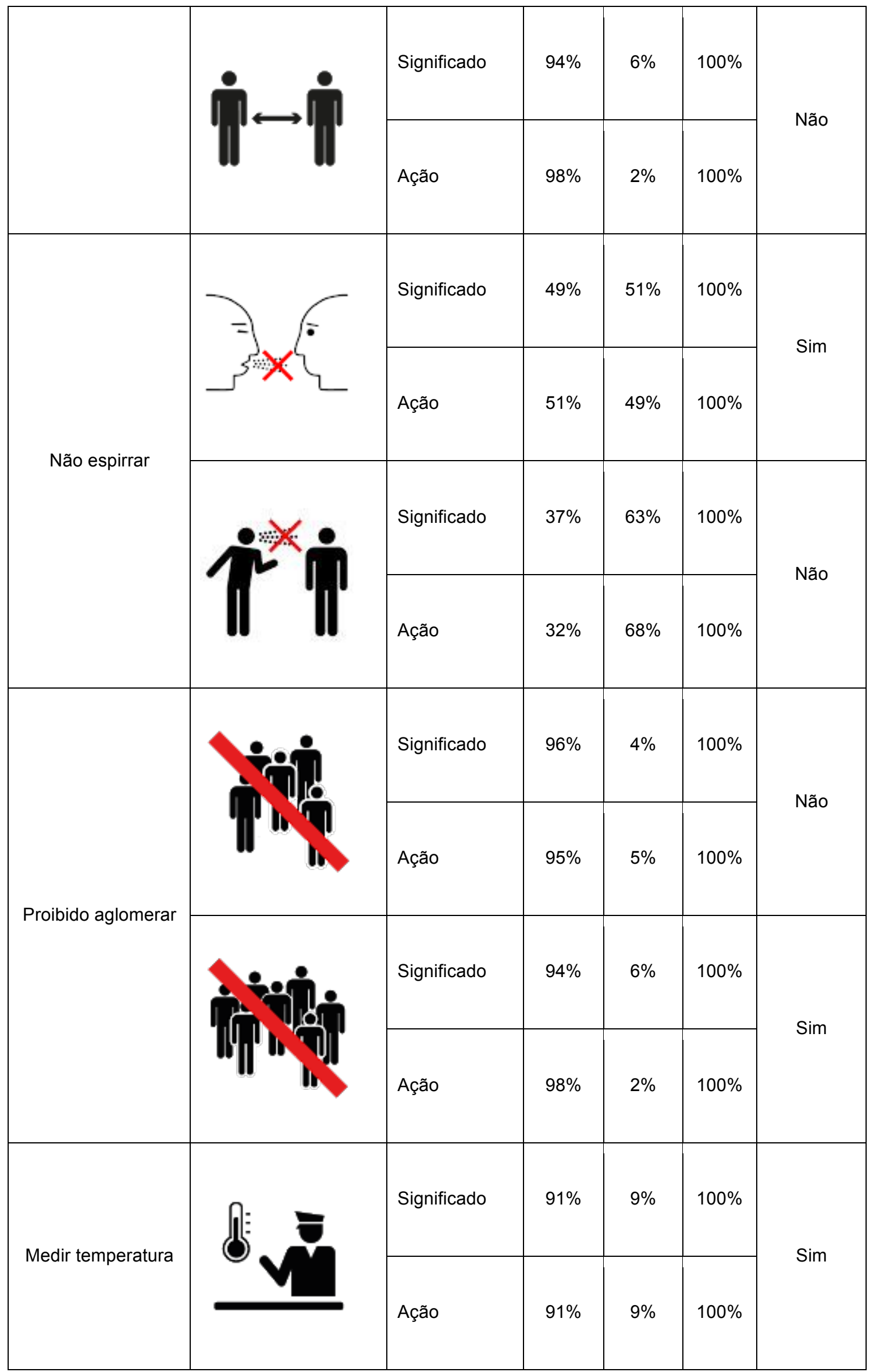




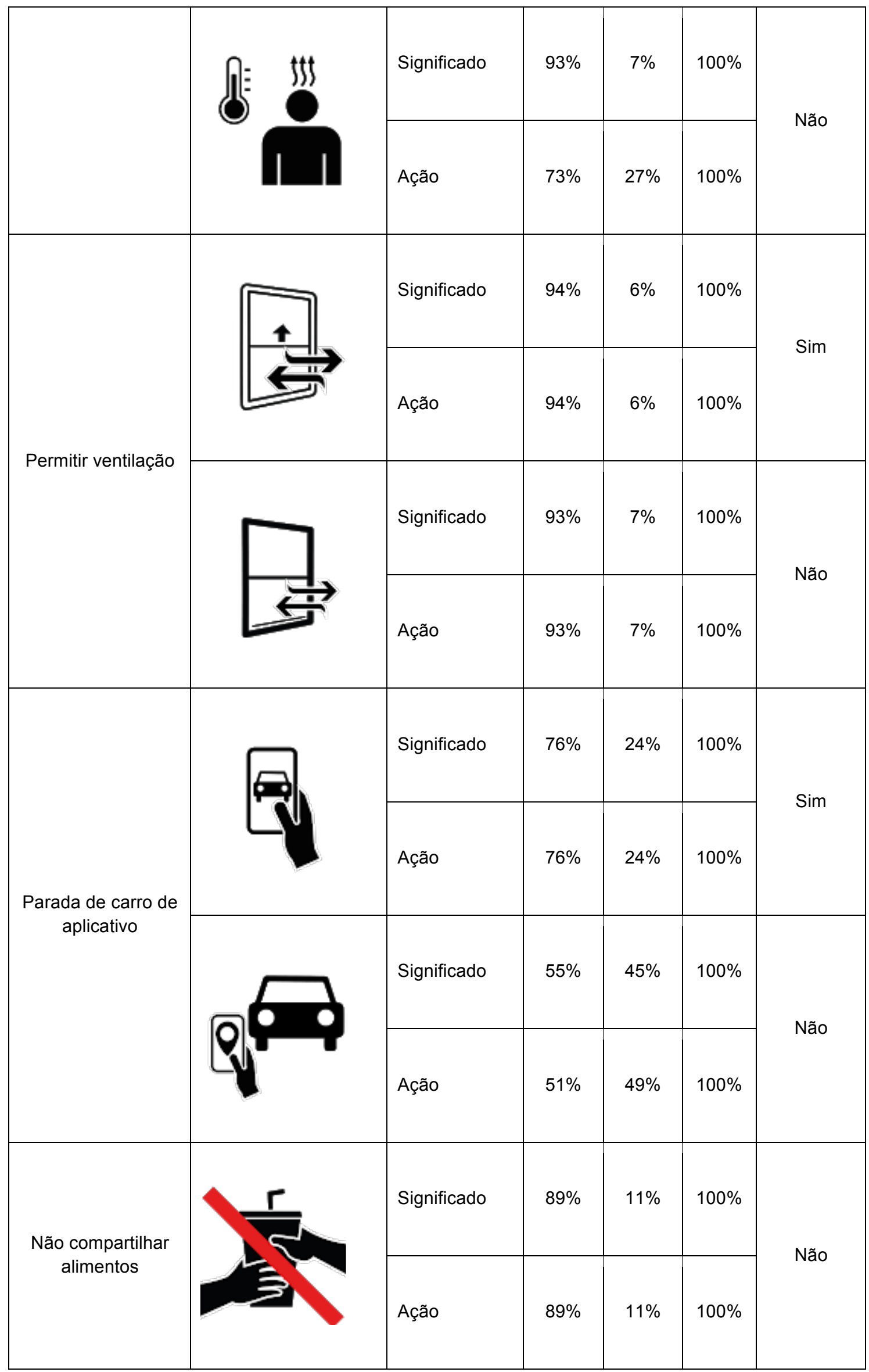




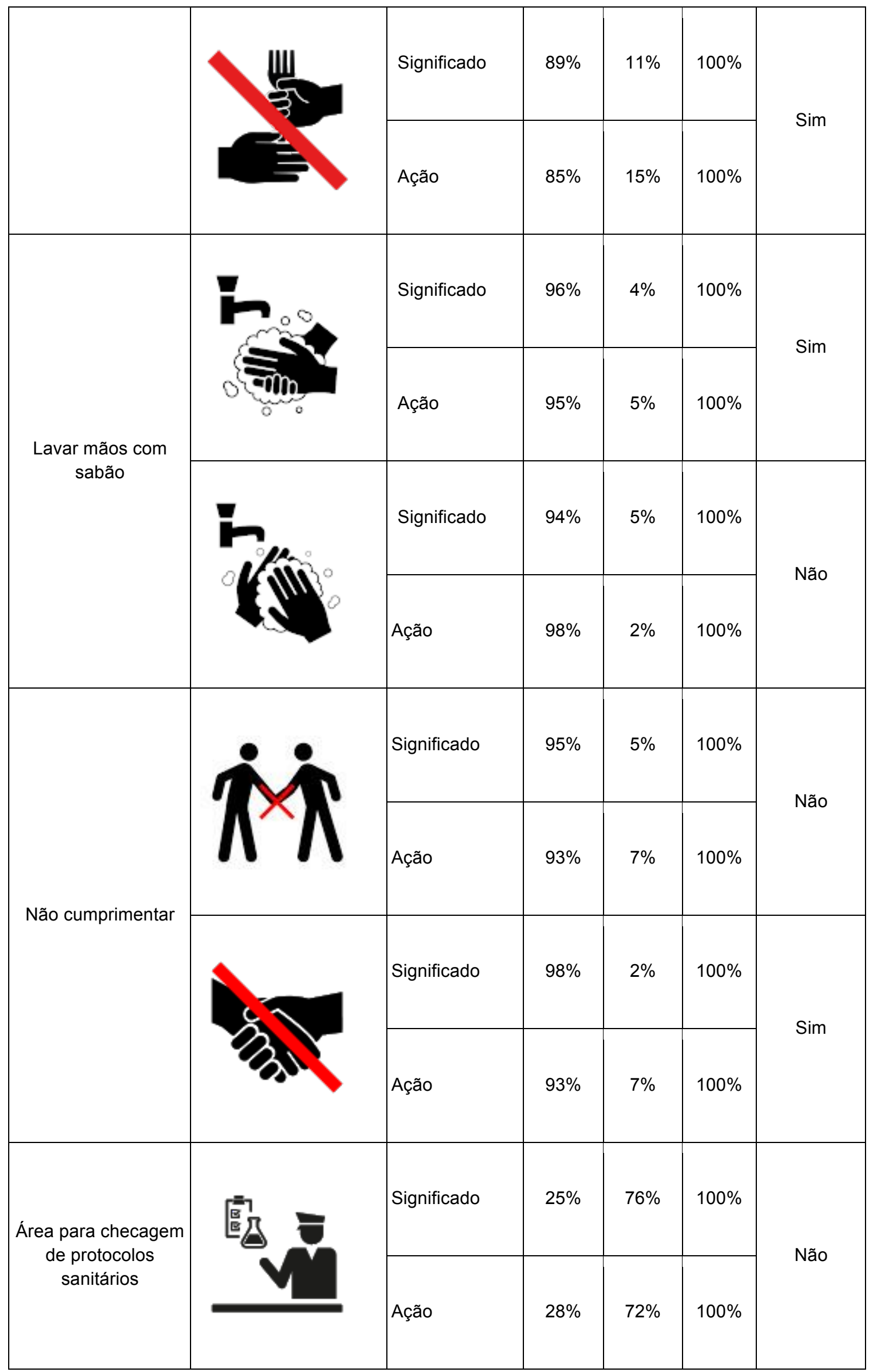




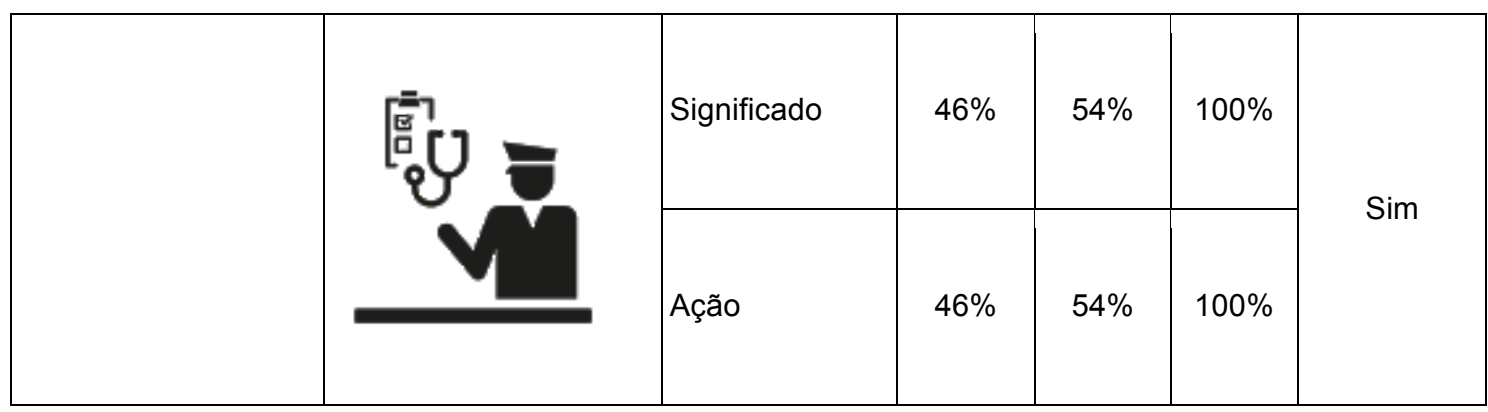

Certas respostas geraram divergências em sua classificação como certa ou errada, explicitando a diversidade de interpretações que uma mensagem pode ter pelos diferentes usuários. O pictograma "não cumprimentar", por exemplo, obteve respostas que não faziam referência ao contato físico, apenas ao ato do cumprimento. Nesse caso, foram consideradas como certas as respostas que apresentaram tais compreensões. Já na mensagem "não tossir/ espirrar nos outros", as respostas que fizeram referência ao ato de conversar alto foram julgadas como erradas, por não considerarem corretamente a principal ação esperada.

Como resultado, grande parte dos pictogramas apresentaram respostas corretas, assertivas e similares. Este resultado provavelmente se deve à familiaridade já existente com esses pictogramas, visto que suas respectivas mensagens são reforçadas constantemente no contexto atual da pandemia e os símbolos elaborados partiram do estudo de pictogramas preexistentes nas normas vigentes (Lesch, et al., 2011).

De acordo com a ISO/TC 145, para um símbolo ser aprovado é necessário atingir $66 \%$ de respostas corretas no teste de compreensibilidade (Foster; Koyama; Adams, 2010). Dos 12 pictogramas selecionados, apenas dois não chegaram ao valor mínimo, "Área para checagem de protocolos sanitários" e "Não espirrar "; ainda assim, símbolos cuja compreensibilidade é menor que $66 \%$ podem ser adotados pela ISO 7001 desde que utilizados com apoio textual, auxiliando no treinamento do público.

\section{Considerações Finais}

Através do desenvolvimento desse projeto, notou-se como muitos dos símbolos gráficos que alcançaram maiores índices de compreensibilidade obtiveram estes resultados devido à semelhança com pictogramas já em uso (Lesch et al., 2011). Nestes casos, este trabalho buscou justamente pesquisar, sintetizar, desenvolver e formalizar a inclusão de uma versão padronizada e graficamente estável destes pictogramas na ISO 7001, onde eles ainda não figuram.

Quanto aos pictogramas que alcançaram menores índices de compreensibilidade (não espirrar, parada de carro de aplicativo e área para checagem de protocolos sanitários), estes representam inovações na linguagem pictográfica e evidenciam, mais uma vez, a importância do papel da ISO na padronização dos símbolos de informação na comunicação, que os torna reconhecíveis e familiares para o público através da sua ampla adoção e divulgação. 


\section{Referências}

Bakker, W. (2013). Icograda and the development of pictogram standards: 1963-1986. Iridescent: Icograda Journal of Design Research.

Duarte, M. E. C., Rosa, C. Rebelo, F e Duarte, C. (2009). Design of pictograms: a comparison between iterative and non-iterative design methodologies. 17th Triennial Congress of the International Ergonomics Association, IEA 2009. Pequim.

Foster, J.; Koyama, K.; Adams, A. (2010). Paper and on-line testing of graphical access symbols in three countries using the ISO 9186 comprehension test. Information Design Journal, 18 (2), pp. 107-117.

Holmes, N. (2018). Pictograms: a view from the drawing board or, what I have learned from Otto Neurath and Gerd Arntz (and Jazz). Information Design Journal, 10 (2), pp. 133-143, 2001.

ISO 7001 Graphical Symbols: Public Information Symbols.

ISO 22727. (2007). Graphical symbols - Creation and design of public information symbols Requirements.

ISO 91860-1. (2018). Graphical Symbols: test methods - Part 1: Methods for testing comprehensibility.

Lesch, M.; Wogalter M.; Powell, W.R.; Horrey, W.J. (2011). Age-related differences in warning symbol comprehension and training effectiveness: Effects of familiarity, complexity, and comprehensibility. Ergonomics 54(10): 879-90.

Moro, M. H. G. (2016). Pictograma e Pictografia: objeto, representação e conceito. UTFPR.

Lupton, E. \& Miller, A. (2011). Design, Escrita e Pesquisa. Porto Alegre: Grupo A.

Pauluk, M. (2020). Pictogramas: História, Design e Normalização. Projeto de Pesquisa. Curitiba: UFPR.

World Health Organization. WHO Director-General's opening remarks at the media briefing on COVID-19. Disponível em

$<$ https://www.who.int/director-general/speeches/detail/who-director-general-s-openingremarks-at-the-media-briefing-on-covid-19---11-march-2020>.

\section{Sobre o(a/s) autor(a/es)}

Mariana S. Lukasinski, estudante, UFPR, Brasil <marianalukasinski@gmail.com> Isabela Amanda Ciuneck, estudante, UFPR, Brasil <i.ciuneck@gmail.com> Eduardo S. Zabloski, estudante, UFPR, Brasil <dustoccox@gmail.com> Ana Carolina S. Costa, estudante, UFPR, Brasil <anacarolinascosta23@hotmail.com> Marcel P. Pauluk, professor, UFPR, Brasil<marcelpauluk@ufpr.br> 